\title{
Semantic Relativism and Logical Implication
}

\author{
Leonid Tarasov ${ }^{1}$ (D)
}

Received: 10 January 2019 / Accepted: 22 October 2020 / Published online: 27 November 2020

(c) The Author(s) 2020

\begin{abstract}
Semantic relativism is the view that the truth-value of some types of statements can vary depending on factors besides possible worlds and times, without any change in their propositional content. It has grown increasingly popular as a semantic theory of several types of statements, including statements that attribute knowledge of a proposition to a subject (knowledge attributions). The ways of knowing claim is the view that perception logically implies knowledge. In my "Semantic Relativism and Ways of Knowing" (2019) I argued that a relativist semantics for knowledge attributions is incompatible with the ways of knowing claim. I suggested that this incompatibility depends on some basic features of the logic of relativist semantics, and therefore can be shown to generalise beyond the discussion of knowledge attributions to semantic relativism more broadly. Here I make this generalisation. I demonstrate that for any proposition $\mathrm{p}$ expressed by a statement that does not have a relativist semantics, and for any proposition q expressed by a statement that does have a relativist semantics, $\mathrm{p}$ fails to logically imply q. I explain why this happens, discuss some of its philosophical consequences, and consider a way to modify relativist semantics to avoid it. I conclude that semantic relativism raises interesting philosophical questions that have gone largely unnoticed in discussions of this view until now.
\end{abstract}

\section{Introduction}

Semantic relativism is now established as a formally rigorous and philosophically robust framework for the analysis of a number of expressions, including predicates of personal taste (e.g. 'good', 'tasty', 'fun'), moral judgement (e.g. 'wrong'), epistemic predicates (e.g. 'know'), and others. It says that the truth-value of statements that contain these expressions can vary depending on factors besides possible worlds and times, without any change in their propositional content. For instance, if Rick and Morty say 'Adventures are fun' to each other, they may say the same thing at the same time, but what they say might be true relative to Rick and false relative to Morty. This will be

Leonid Tarasov

leonid.tarasov@manchester.ac.uk; leonidvtarasov@outlook.com

1 The University of Manchester, Oxford Road, Manchester M13 9PL, UK 
made more precise in Section 2, but roughly the idea is that, besides the possible world and time of the utterance, the truth-value of 'Adventures are fun' is sensitive to a standard of fun that is different for different people. This works in a similar way for 'wrong', 'know', and other expressions, except here the sensitivity is to standards of morality, epistemic standards, and so on (see, e.g., Kölbel 2008; MacFarlane 2014).

In a recent paper (2019), I argued that relativism about 'know' is incompatible with the ways of knowing claim. The ways of knowing claim is the claim that for any subject $\mathrm{x}$ and any proposition $\mathrm{p}$, the proposition that $\mathrm{x}$ perceives that $\mathrm{p}$ (call this the 'perception proposition') logically implies the proposition that $\mathrm{x}$ knows that $\mathrm{p}$ (call this the 'knowledge proposition'). For example, that Danny saw that Humpty Dumpty had a fall logically implies that Danny knows that Humpty Dumpty had a fall. I already gave (e.g. 2019: 2103-2104) a number of reasons to think that the ways of knowing claim is significant, and the emphasis in the present paper is on generalising my findings, so I won't defend the ways of knowing claim. For our present purposes it should suffice to note that it enjoys an unusually long philosophical pedigree: from Russell (1948: 422), Moore (1953: 77), Chisholm (1989: 41), to Williamson (2000: 34).

I noted (2019: 2090) that this incompatibility is a consequence of the logic of semantic relativism, by which I mean the set of formal principles that underlie relativist semantics; for example, I noted that the incompatibility can be demonstrated in purely set theoretic terms, with minimal assumptions about how set membership is determined and no assumptions about the members themselves. I will explain the incompatibility in detail later on, but at root the problem arises because the truth-value of the knowledge proposition is sensitive to a standard that the perception proposition is not sensitive to.

I did suggest (2019: 2104) that this kind of incompatibility generalises beyond 'know' and the ways of knowing claim to semantic relativism more broadly. However, I did not demonstrate this and only briefly discussed some of its implications.

Here I demonstrate that the incompatibility does generalise to semantic relativism more broadly and discuss some broader implications of this generalisation. The paper is structured into seven sections. In Sect. 2 I outline the relevant aspects of relativist semantics and define logical implication. In Sect. 3 I generalise my earlier claims without reference to any concrete examples. No examples are used in order to show that the claims do not depend on the details of a given debate, but on some basic conditions that can occur as a consequence of the logic of the relativist framework. In Sect. 4 I provide a few concrete examples. In Sects. 5 and 6 I discuss two philosophical consequences of semantic relativism that arise as a result of the above discussion. In Sect. 7 I consider a way to modify relativist semantics to avoid these consequences. I conclude in Sect. 8.

\section{Relativist Semantics}

\subsection{Circumstances of Evaluation and Relativism}

The foundation for relativist semantics is usually David Kaplan's well-known "Demonstratives" (1989). In Kaplan's framework sentences in contexts (i.e. sentence types 
tokened through use in speech or writing) express propositions or contents that are evaluated for truth at so-called circumstances of evaluations, which are characterised as ordered pairs of possible worlds and times. ${ }^{1}$ For shorthand, we will use 'sentence' to mean 'sentence in context' in what follows. For instance, suppose a sentence $\Phi$ expresses the proposition that $\mathrm{p}$; the truth-value of $\mathrm{p}$ is determined as a function of a circumstance $<\mathrm{w}, \mathrm{t}>$, where the parameters $\mathrm{w}$ and $\mathrm{t}$ stand for a possible world and a time.

Relativists extend this framework in one of two ways. Moderate relativists like Max Kölbel (e.g. 2008) say that there are parameters of circumstances besides w and $t$ and the values of these unorthodox parameters are determined by the context in which the sentence was tokened, i.e. the context of use or utterance. Radical relativists like John MacFarlane (e.g. 2014) also say that there are unorthodox parameters, but the values of both orthodox and unorthodox parameters may be determined by so-called contexts of assessment, which may or may not overlap with the contexts of use. Contexts of assessment are understood to represent a possible situation in which the utterance of a sentence might be evaluated for truth.

For example, both moderate and radical relativists would say that sentence (1) expresses the proposition ADVENTURE:

(1) Adventures are fun.

ADVENTURE: that adventures are fun.

And they would say that the truth-value of ADVENTURE varies as a function of the value of a standards of taste parameter $\mathrm{f}$. When evaluating uttered sentences, moderate relativists would index $\mathrm{f}$ to the context of utterance, and radical relativists would index $\mathrm{f}$ to the context of assessment. ${ }^{2}$ For instance, let's say Rick likes adventures and Morty hates them. Then according to moderate relativism, Rick's context of utterance sets a value of $f$ such that ADVENTURE comes out true, and Morty's such that ADVENTURE comes out false. According to radical relativism, Rick's context of assessment sets a value of $\mathrm{f}$ such that ADVENTURE comes out true, and Morty's such that ADVENTURE comes out false. When the context of utterance and the context of assessment overlap, moderate and radical relativism yield the same truth-values. The difference between the two views is revealed when the context of utterance and the context of assessment come apart. Suppose that Rick utters 'Adventures are fun' and both Rick and Morty evaluate whether what Rick is said is true or false. Following moderate relativism, what Rick said is true for both Rick and Morty; the truth-value is

\footnotetext{
${ }_{1}^{1}$ I am grateful to an anonymous reviewer for reminding me that while Kaplan (1989: 500) acknowledges that 'the content of a sentence in a given context is what has traditionally been called a proposition', he also explicitly notes that for him 'a content [is] a notion applying not only to sentences taken in a context but to any meaningful part of speech taken in a context' (Kaplan 1989: 501). In other words, the notion of content is broader than the notion of proposition. In this paper it will do no harm to equate the two.

${ }^{2}$ Here I ignore the thought that the values of the orthodox parameters $\mathrm{w}$ and $\mathrm{t}$ can also be determined by contexts of assessment. It is not relevant to our purposes and would complicate the semantics we are working with. See MacFarlane (2014: 49-52) for discussion. I am grateful to an anonymous reviewer for reminding me that the unorthodox parameter does not have to be a standard, but could be a judge or an assessor (e.g. Glanzberg 2007). The discussion above could be modified to work with an alternative parameter (see Tarasov 2014: Ch. 5).
} 
a function of Rick's context of utterance and Rick likes adventures. In contrast, following radical relativism, what Rick said is false for Morty; the truth-value is a function of Morty's context of assessment and Morty hates adventures. But it is true for Rick; the truth-value is a function of Rick's context of assessment.

We need not dwell on these differences. What is important here is that for both types of relativism, a circumstance of evaluation in this case is a tuple $<\mathrm{w}, \mathrm{t}, \mathrm{f}>$. More generally, both moderate and radical relativists add unorthodox parameters to circumstances and - this last part is crucial for the demonstration in $\S 3$ belowthe values of the parameters of circumstances vary independently of each other. To make things easier in the rest of the paper, let us symbolise the unorthodox parameter with n. Finally, because what we will say does not depend on variation in times, for the sake of simplicity let us drop these from circumstances.

Thus, we will understand circumstances of evaluation just as pairs $<w, n>$, where the values of $w$ and $n$ vary independently of each other. The truth-value of the proposition expressed by a sentence is evaluated with respect to the values of $\mathrm{w}$ and $\mathrm{n}$.

\subsection{Assessment-Sensitivity and Insensitivity}

According to semantic relativism, all sentences express propositions that are evaluated with respect to $<w, n>$. However, not all propositions are evaluated in the same way. Sentences like (1) express propositions whose truth-values vary when the values of either $w$ or $n$ vary. Other sentences express propositions whose truth-values vary only when the value of $w$ varies; changes in the value of $n$ make no difference to the truth-value of the proposition. Our formal semantic system looks at the values of all the parameters to yield a truth-value for any proposition, but in the case of propositions expressed by the latter type of sentences, the value of $n$ makes no practical difference to their truth-values. Semantic relativism is not, or need not be, a form of global relativism (see Wright 2008: 166-68).

MacFarlane (2014: 90) calls the two types of sentences assessment-sensitive and assessment-insensitive (see also Tarasov 2019: 2092):

$A S$ : A sentence $\Phi$ is assessment-sensitive if and only if there is a circumstance of evaluation $<\mathrm{w}_{1}, \mathrm{n}_{1}>$ at which the proposition expressed by $\Phi$ is true, and there is another circumstance of evaluation $<\mathrm{w}_{1}, \mathrm{n}_{2}>$ at which it is false, where $\mathrm{n}_{2} \neq \mathrm{n}_{1}$.

AIS: A sentence $\Phi$ is assessment-insensitive if and only if, if the proposition expressed by $\Phi$ is true at circumstance $<\mathrm{w}_{1}, \mathrm{n}_{1}>$, then it is also true at every circumstance which is a member of the set $\left\{\left\langle\mathrm{w}_{1}, \mathrm{n}_{2}\right\rangle,\left\langle\mathrm{w}_{1}, \mathrm{n}_{3}\right\rangle, \ldots,<\mathrm{w}_{1}\right.$, $n_{n}>$ \}, where $n_{n}$ is any value of the parameter $n .^{3}$

\footnotetext{
3 In other words, if we hold the value of the worlds parameter w fixed, in the case of $A S$ we will find at least one value of $\mathrm{n}$ such that the proposition expressed by $\Phi$ is true and another value of $\mathrm{n}$ such that the proposition is false. Conversely, if we hold the value of the worlds parameter w fixed, in the case of AIS, we will find that changes in the value of $n$ make no difference to the truth-value of the proposition; the proposition is true or false at all values of $\mathrm{n}$. I am grateful to an anonymous reviewer for pressing me to tidy up the formal definitions $A S$ and $A I S$ (any lingering technical issues are my own).
} 
We have already seen an example of a sentence that fits AS, viz. sentence (1). Here is an example of a sentence that fits AIS:

(2) Rick built a gun.

Presumably, whether Rick built a gun is not dependent on a context of assessment; it is intuitive that this is not something dependent on someone's judgement or standard. The present framework allows us to respect this intuition; (2) can be analysed as assessment-insensitive.

An important point to emphasize here is that, if we evaluate a proposition that is expressed by an assessment-sensitive sentence, we will find at least one value of $\mathrm{n}$ at which that proposition is false; otherwise, the sentence would not be assessment-sensitive after all. I have noted (2019: 2095) that relativists make an analogy between the way contingent sentences are modelled in possible world semantics and assessment-sensitive sentences are modelled in relativist semantics: just as in a possible worlds semantic model for contingent sentences there must be at least one possible world at which the proposition expressed by a contingent sentence is true and at least one possible world at which the proposition is false, so in a semantic model for assessment-sensitive sentences there must be at least one value of a parameter fixed by a context of assessment with respect to which the proposition expressed by an assessment-sensitive sentence is true and at least one value of this parameter with respect to which the proposition is false.

As we go along, the interaction between propositions along the two axes of circumstances of evaluation will be made clearer with the use of concrete examples presented within toy semantic models that include as few circumstances of evaluation as possible. This initial exposition is here to give a general sense of the fine workings of the relativist system through a familiar analogy with possible worlds.

\subsection{Logical Implication}

Finally, we need to define logical implication. I have previously extended (2019: 2094) MacFarlane's (2014: 68) definition of logical implication from sentences and open formulas to propositions as follows:

Logical Implication: A proposition p is logically implied by a set Q of propositions if and only if for every circumstance of evaluation $j$, if every member of $Q$ is true at $\mathrm{j}$, then $\mathrm{p}$ is true at $\mathrm{j}$.

We now have enough to generalise my earlier claims. I note at the outset that the next two sections borrow heavily from my earlier work, but I will omit further reference to it to keep things tidy. I also note that the finer technical details, including justification for a number of simplifications in the presentation of relativist semantics above, are given in my earlier paper, especially in Appendix 1 of that paper. These details are not needed to make the arguments in this paper work, but they should reassure us that these arguments do not go through simply because I have omitted or misrepresented crucial technical elements in my account. 
Table 1 Truth-table for the material conditional as defined in classical two-valued logic

\begin{tabular}{lll}
\hline $\mathrm{p}$ & $\mathrm{q}$ & $\mathrm{p} \supset \mathrm{q}$ \\
\hline $\mathrm{T}$ & $\mathrm{T}$ & $\mathrm{T}$ \\
$\mathrm{T}$ & $\mathbf{F}$ & $\mathrm{F}$ \\
$\mathrm{F}$ & $\mathbf{T}$ & $\mathrm{T}$ \\
$\mathrm{F}$ & $\mathbf{F}$ & $\mathrm{T}$ \\
\hline
\end{tabular}

\section{When p does not Logically Imply q}

\subsection{Assessment-Sensitive and Insensitive Sentences}

To begin with, note that the definition of logical implication given by relativists, cited in Sect. 2.3 above, is mutatis mutandis equivalent to the definition of the material conditional in propositional logic. Thus, the familiar truth-table for the material conditional in classical two-valued logic might be helpful here (see Table 1). Specifically, it might be helpful to note that all the examples we are interested in below are ones that would fall on the second row in the truth-table, i.e. examples where the antecedent is true, the consequent is false, and therefore the material conditional is false. We are not interested at all in the other types of cases and ignore them in what follows. Finally, note that full proofs for the points made below are given in the Appendix.

Suppose that $\mathrm{p}$ is expressed by an assessment-insensitive and contingent sentence. Suppose that $\mathrm{q}$ is expressed by an assessment-sensitive and contingent sentence. Imagine too that we are working in a toy semantic model that has four, and only four, circumstances of evaluation: $<\mathrm{w}_{1}, \mathrm{n}_{1}>,<\mathrm{w}_{1}, \mathrm{n}_{2}>,<\mathrm{w}_{2}, \mathrm{n}_{1}>$ and $<\mathrm{w}_{2}$, $\mathrm{n}_{2}>$.

Now assume that $\mathrm{q}$ is true at $<\mathrm{w}_{1}, \mathrm{n}_{1}>$. Then, following AS above, q must be false at every other circumstance of evaluation in our toy model. That is, since $q$ is expressed by an assessment-sensitive sentence and is true at $\mathrm{n}_{1}$, $\mathrm{q}$ has to be false at $\mathrm{n}_{2}$; otherwise, $\mathrm{q}$ would be true at every value of $\mathrm{n}$ and would therefore be expressed by a sentence that is not assessment-sensitive after all. And since the sentence in question is also contingent and $\mathrm{q}$ is true at $\mathrm{w}_{1}$, $\mathrm{q}$ has to be false at $\mathrm{w}_{2}$; otherwise, $\mathrm{q}$ would be true at every value of $\mathrm{w}$ and would therefore be expressed by a sentence that is not contingent after all. Together, this is every combination of the values of $\mathrm{w}$ and $n$ in our model.

Assume that $\mathrm{p}$ is true at $\left\langle\mathrm{w}_{1}, \mathrm{n}_{1}\right\rangle$. Then, following $A I S$ above, $\mathrm{p}$ must also be true at $<\mathrm{w}_{1}, \mathrm{n}_{2}>$ and false at every other circumstance of evaluation in our toy model. That is, since $\mathrm{p}$ is expressed by an assessment-insensitive sentence and is true at $n_{1}$, p has to be true at $n_{2}$; otherwise, $p$ would be true at some values of $n$ and false at others and would therefore be expressed by a sentence that is not assessment-insensitive after all. Similarly, since the sentence in question is also contingent and $\mathrm{p}$ is true at $\mathrm{w}_{1}, \mathrm{p}$ has to be false at $\mathrm{w}_{2}$; otherwise, $\mathrm{p}$ would be true at every value of $\mathrm{w}$ and would therefore be expressed by a sentence that is not contingent after all. Together, this is every combination of the values of $w$ and $n$ in our model. 
Table 2 Circumstances of evaluation at which $\mathrm{p}$ and $\mathrm{q}$ are true and circumstances at which they are false - Case 1

\begin{tabular}{lll}
\hline & w $_{\mathbf{1}}$ & $\mathbf{w}_{\mathbf{2}}$ \\
\hline $\mathrm{n}_{1}$ & $\mathrm{p}, \mathrm{q}$ \\
$\mathrm{n}_{2}$ & $\mathrm{p}$ & \\
\hline
\end{tabular}

Notice that there is one circumstance of evaluation, namely $\left\langle\mathrm{w}_{1}, \mathrm{n}_{2}\right\rangle$, in our toy model at which $\mathrm{p}$ is true but $\mathrm{q}$ is false, as shown in Table 2 below. ${ }^{4}$

Following the definition of logical implication in $\$ 2.3$, to say that there is at least one circumstance of evaluation at which $\mathrm{p}$ is true but $\mathrm{q}$ is false is just to say that $\mathrm{p}$ does not logically imply q. For convenience, the toy semantic model has only four circumstances, but we should be able to see that this result holds for any value of $\mathrm{w}$ and $\mathrm{n}^{5}$

\subsection{Assessment-Sensitive Sentences Only}

Suppose that $\mathrm{p}$ and $\mathrm{q}$ are both expressed by assessment-sensitive sentences, where the truth-value of $p$ changes with respect to changes in the value of an unorthodox parameter $n$, but the truth-value of $q$ with respect to another unorthodox parameter

\footnotetext{
${ }^{4}$ For a given circumstance of evaluation, read down from the worlds columns ( $\mathrm{w}_{1}{ }^{\prime}$, ' $\mathrm{w}_{2}{ }^{\prime}$ ) and across from the non-orthodox parameter rows $\left({ }^{\prime} n_{1}{ }^{\prime},{ }^{\prime} n_{2}{ }^{\prime}\right)$; the circumstances at which $\mathrm{p}$ and $\mathrm{q}$ are true are marked with 'p' and 'q' respectively.

5 I am grateful to an anonymous reviewer for highlighting the following complication in this argument (and the analogous argument in §3.2). Suppose that $w_{1}$ is a possible world in which adventures raise adrenaline and $\mathrm{w}_{2}$ is a possible world in which adventures lower adrenaline. Suppose that $\mathrm{n}_{1}$ is a standard according to which raised adrenaline is fun and lowered adrenaline is not fun, and $\mathrm{n}_{2}$ is the reverse standard. Finally, suppose that 'Adventures are fun' is assessment-sensitive and that $\left\langle\mathrm{w}_{1}, \mathrm{n}_{1}\right\rangle,\left\langle\mathrm{w}_{1}\right.$, $\mathrm{n}_{2}>,<\mathrm{w}_{2}, \mathrm{n}_{1}>$ and $<\mathrm{w}_{2}, \mathrm{n}_{2}>$ are all the circumstances of evaluation there are. Then the proposition ADVENTURE is true at $<\mathrm{w}_{1}, \mathrm{n}_{1}>$ and at $<\mathrm{w}_{2}, \mathrm{n}_{2}>$, and false at $<\mathrm{w}_{1}, \mathrm{n}_{2}>$ and $<\mathrm{w}_{2}, \mathrm{n}_{1}>$. So here ADVENTURE does not behave like the proposition $q$ in Table 2. I confess I am not sure whether this is a genuine counter-example to my argument or merely a limiting case. Firstly, note that it leaves no room for presupposition failure: adventures raise adrenaline in some possible worlds, and lower it in others, but adventures are present in all possible worlds, which is odd. Secondly, notice that the example makes an assumption about the relationship between a physical fact and a standard of taste, viz. that raised/ lowered adrenaline is fun/not fun. Because of this, the assumption is theoretically loaded. More importantly, it is unclear to me whether it smuggles something in that begs the question against our argument. For example, suppose I want to say that ADVENTURE is logically implied by ADVENTURE*, where ADVENTURE* $^{*}$ is the proposition that adventures raise adrenaline. What should I say about this if the standards of fun are already said to respond to raised or lowered adrenaline? Presumably, I would have to say that the fact that adventures raise adrenaline does not logically imply that adventures are fun, because we have already assumed that the 'fun factor' of adventures is responsive to raised adrenaline for some and lowered adrenaline for others. Be that as it may, even if this is a counter-example to the letter of the argument as I have set it out above, I am also grateful to the anonymous reviewer for noting that it is not a counter-example to the overall thrust of the argument. Even in the present example, we can assume that there is at least one circumstance at which $\mathrm{p}$ is true and ADVENTURE is false, viz. either at $<\mathrm{w}_{1}, \mathrm{n}_{2}>$ or at $\left\langle\mathrm{w}_{2}, \mathrm{n}_{1}\right\rangle$. More generally, the reviewer has very generously suggested a general proof of the point I am trying to make here, which I set out in the Appendix.
} 
Table 3 Circumstances of evaluation at which $\mathrm{p}$ and $\mathrm{q}$ are true and circumstances at which they are false - Case 2

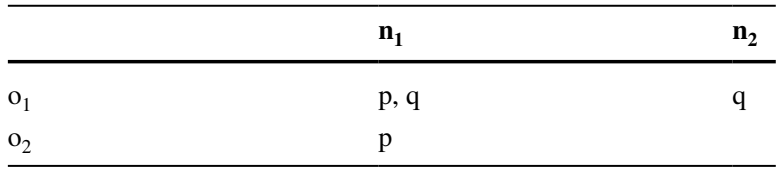

o. The truth-value of $\mathrm{p}$ does not change with respect to o, and the truth-value of $\mathrm{q}$ does not change with respect to $\mathrm{n}$. Call this being assessment-sensitive with respect to $n$ and with respect to o respectively.

We now need to characterise circumstances of evaluation as tuples $<\mathrm{w}, \mathrm{n}, \mathrm{o}\rangle$, where the values of these parameters vary independently of each other. However, the value of the possible worlds parameter $\mathrm{w}$ makes no difference to our argument, so we will drop it from our example to keep thing as simple as possible. For the sake of the argument, we will assume that, if $\mathrm{p}$ or $\mathrm{q}$ are true, they are true at all possible worlds. Thus, we will take circumstances to be pairs $\langle\mathrm{n}, \mathrm{o}\rangle$, where the values of $n$ and o vary independently of each other. ${ }^{6}$

Finally, imagine that we are working in a toy semantic model that has four, and only four, circumstances of evaluation: $<\mathrm{n}_{1}, \mathrm{o}_{1}>,<\mathrm{n}_{1}, \mathrm{o}_{2}>,<\mathrm{n}_{2}, \mathrm{o}_{1}>$ and $<\mathrm{n}_{2}$, $\mathrm{O}_{2}>$.

Now assume that $\mathrm{q}$ is true at $\left\langle\mathrm{n}_{1}, \mathrm{o}_{1}\right\rangle$. Then $\mathrm{q}$ must also be true at $\left.<\mathrm{n}_{2}, \mathrm{o}_{1}\right\rangle$ and false at every other circumstance of evaluation in our toy model. That is, since $q$ is expressed by a sentence that is assessment-sensitive with respect to o but not $\mathrm{n}$ and is true at $n_{1}$, $q$ has to be true at $n_{2}$. Otherwise, $q$ would be true at some values of $n$ and false at others and would therefore be expressed by a sentence that is assessment-sensitive with respect to $\mathrm{n}$ after all. Similarly, since $\mathrm{q}$ is expressed by a sentence that is assessment-sensitive with respect to o and is true at $\mathrm{o}_{1}, \mathrm{q}$ has to be false at $\mathrm{O}_{2}$. Otherwise, $\mathrm{q}$ would be true at all values of $\mathrm{o}$ and would therefore be expressed by a sentence that is not assessment-sensitive with respect to o after all. Together, this is every combination of the values of $n$ and o in our model.

Assume that $\mathrm{p}$ is true at $\left\langle\mathrm{n}_{1}, \mathrm{o}_{1}\right\rangle$. Then $\mathrm{p}$ must also be true at $\left\langle\mathrm{n}_{1}, \mathrm{o}_{2}>\right.$ and false at every other circumstance of evaluation in our toy model. That is, since $p$ is expressed by a sentence that is assessment-sensitive with respect to $\mathrm{n}$ but not $\mathrm{o}$ and is true at $o_{1}, p$ has to be true at $o_{2}$. Otherwise, $p$ would be true at some values of $o$ and false at others and would therefore be expressed by a sentence that is assessment-sensitive with respect to o after all. Similarly, since $\mathrm{p}$ is expressed by a sentence that is assessment-sensitive with respect to $n$ and is true at $n_{1}$, $p$ has to be false at $n_{2}$. Otherwise, $p$ would be true at all values of $n$ and would therefore be expressed by a sentence that is not assessment-sensitive with respect to $\mathrm{n}$ after all.

\footnotetext{
${ }^{6}$ Alternatively, we could keep the parameter $w$ in our characterisation of circumstances and just hold the value of this parameter fixed, varying the values of o and $n$ only. This would achieve the same conclusion, but it would be messier and complicate Table 3.
} 
Now notice that there is one circumstance of evaluation, namely $\left\langle\mathrm{n}_{1}, \mathrm{o}_{2}\right\rangle$, in our toy model at which $\mathrm{p}$ is true but $\mathrm{q}$ is false, as shown in Table 3 below.

Once again, $\mathrm{p}$ fails to logically imply q. ${ }^{7}$

\subsection{General Conditions for $p$ to Fail to Logically Imply q}

The demonstration that $\mathrm{p}$ does not logically imply $\mathrm{q}$ in the previous two sub-sections is quite general: we have said nothing about the content of propositions $\mathrm{p}$ and q, nor about particular expressions that are supposed to be good candidates for a relativist analysis. The result that $\mathrm{p}$ does not logically imply $\mathrm{q}$ simply falls out of our characterisation of circumstances of evaluation, the interaction between different parameters of the circumstances and the definition of logical implication, all of which are prescribed by relativists. In other words, the result that $\mathrm{p}$ does not logically imply $\mathrm{q}$ is a consequence of the logic that underlies relativist semantics. This can be expressed in terms of two general conditions (A) and (B).

$\mathrm{p}$ does not logically imply $\mathrm{q}$ if $\mathrm{A}$ and $\mathrm{B}$ are true.

(A) The truth-value of the proposition q varies with respect to a parameter of circumstances of evaluation, such that there is at least one value of this parameter at which $q$ is true and at least one value at which $q$ is false.

(B) The truth-value of the proposition $\mathrm{p}$ does not vary with respect to this parameter.

Following the reasoning in Sect. 3.1, A and B occur whenever p is expressed by an assessment-insensitive sentence and $\mathrm{q}$ is expressed by an assessment-sensitive sentence. Specifically, we saw that $\mathrm{q}$ is true at some values of $\mathrm{n}$ and false at others. In contrast, if $\mathrm{p}$ is true, $\mathrm{p}$ is true at all values of $\mathrm{n}$. Following the reasoning in Sect. 3.2, A and B also occur whenever $\mathrm{p}$ and $\mathrm{q}$ are expressed by assessment-sensitive sentences, provided the truth-values of $\mathrm{p}$ and $\mathrm{q}$ vary with respect to different parameters of circumstances and the values of these parameters vary independently of each other. Specifically, we saw that if $q$ is true, then $q$ is true at all values of $n$, true at some values of $o$ and false at others. In contrast, if $p$ is true, then $p$ is true at all values of $o$, true at some values of $n$ and false others.

$\mathrm{A}$ and $\mathrm{B}$ are true if either $\mathrm{C}$ or $\mathrm{D}$ is true.

(C) p is expressed by an assessment-insensitive and q is expressed by an assessmentsensitive sentence.

(D) $\mathrm{p}$ and $\mathrm{q}$ are expressed by assessment-sensitive sentences, but $\mathrm{p}$ and $\mathrm{q}$ are assessment-sensitive with respect to different parameters of circumstances of evaluation and the values of these parameters vary independently of each other.

\footnotetext{
7 See the Appendix for a general proof of this. I am very grateful to an anonymous reviewer for suggesting the proof.
} 
Thus, we find that $\mathrm{p}$ does not logically imply $\mathrm{q}$ whenever either $\mathrm{C}$ or $\mathrm{D}$ is true.

\section{Some Concrete Examples}

So far we have largely abstracted away from concrete examples of sentences or propositions. We needed to demonstrate that any proposition $\mathrm{p}$ does not logically imply any proposition $\mathrm{q}$ if conditions $\mathrm{A}$ and $\mathrm{B}$ are met, and conditions $\mathrm{A}$ and $\mathrm{B}$ are met whenever either $\mathrm{C}$ or $\mathrm{D}$ is true. We can now start plugging some concrete examples in place of $\mathrm{p}$ and $\mathrm{q}$ to show that this is not only a general feature of relativism, but also a philosophically interesting one.

I will appeal to four pairs of propositions here ${ }^{8}$; call them Perception Pair (see Tarasov 2019: 2094), and Memory, Morality and Skill Pairs. In each pair, the first proposition is plugged in place of $\mathrm{p}$ and the second in place of $\mathrm{q}$, as per the conditions discussed in Sect. 3 above.

A factive verb is any verb that entails the truth of the proposition it takes as its object: 'recognise', 'realise', 'regret', 'remember' and others. For instance, that Justin regretted that Humpty Dumpty had a fall logically implies that Humpty Dumpty had a fall. Note that, following Timothy Williamson (2000: 34), the Memory Pair below works with any factive verb plugged in place of 'remembered', e.g. that Adam regretted that Humpty Dumpty had a fall, that Adam recognised that Humpty Dumpty had a fall, that Adam realised that Humpty Dumpty had a fall, etc. - following Willaimson, all logically imply that Adam knows that Humpty Dumpty had a fall.

Perception Pair:

$\mathrm{PER}_{1}$ : that Danny saw that Humpty Dumpty had a fall.

$\mathrm{PER}_{2}$ : that Danny knows that Humpty Dumpty had a fall.

Memory Pair:

$\mathrm{MEM}_{1}$ : that Adam remembered that Humpty Dumpty had a fall.

$\mathrm{MEM}_{2}$ : that Adam knows that Humpty Dumpty had a fall.

Morality Pair:

MORAL $_{1}$ : that Gordon murdered someone.

$\mathrm{MORAL}_{2}$ : that Gordon did something wrong.

Skill Pair:

$\mathrm{SKILL}_{1}$ : that Jesse is an Olympian.

SKILL $_{2}$ : that Jesse is a good athlete.

It seems intuitive that in each pair the first proposition logically implies the second. For example, it is intuitive that that Gordon murdered someone logically implies

\footnotetext{
${ }^{8}$ I am grateful to several anonymous reviewers for encouraging me to rethink my examples (the pairs used in earlier drafts were much less plausible).
} 
that he did something wrong. ${ }^{9}$ However, since 'know', 'wrong' and 'good' are all supposed to be assessment-sensitive according to relativism, and 'see', 'remember', 'murder' and 'Olympian' are plausibly assessment-insensitive or sensitive to a different parameter, each pair satisfies conditions A and B above. ${ }^{10}$ Therefore, if we adopt relativism, in each pair the first proposition fails to logically imply the second.

This shows that my earlier arguments regarding the incompatibility of the ways of knowing claim and relativism can be generalised not only in abstraction, as shown in $\$ 3$ above, but also in application to concrete examples besides the Perception Pair. This makes for an uncomfortable consequence for semantic relativism. One of the primary motivations for semantic relativism comes from its apparent ability to account for our intuitions about sentences containing expressions like 'know', 'wrong' and 'good' - to assign the semantic value True to these sentences when they seem true to us, and the semantic value False when they seem false (e.g. MacFarlane 2014: 21-22). We now find that relativism runs contrary to our intuitions with respect to pairs of propositions like Perception, Memory, Morality and Skill - our intuition tells us that $\mathrm{PER}_{1}$ logically implies $\mathrm{PER}_{2}, \mathrm{MORAL}_{1}$ logically implies $\mathrm{MORAL}_{2}$, and so on, but relativism cannot support this intuition. So one of the consequences of relativism runs in tension with one of its main motivations.

These conclusions are also suggestive of two broader issues with semantic relativism. Let us call the first commitment to deferentialist methodological priority and the second unexpected relativism, and take them in turn.

\section{Deferentialist Methodological Priority}

There is a view that it is bad philosophical practice to have one's semantics settle one's philosophical theories. That is, some substantive philosophical claim, or the answer to some substantive philosophical question, should not simply fall out of one's semantics for a given set of expressions. Instead, when deciding which philosophical theory to adopt, other factors should also be taken into account, such as explanatory power, consistency, simplicity and so on. There is no consensus as to which or how many factors, nor what weight should be assigned to them, but nonetheless it is thought that they should be taken into account. Chris Daly and David Liggins (2011) put the point as follows:

An attitude of uncritical deference to any non-philosophical discipline is badly misguided. Although the evidence drawn from some non-philosophical disci-

\footnotetext{
${ }^{9}$ Suppose Gordon murdered Dr Breen, and Dr Breen was a tyrant. Is it still intuitive that Gordon did something wrong? I think that it is and intuitions to the contrary could be explained; for example, we could say that it sometimes right to remove a tyrant, and so one can sometimes be exonerated for murdering, but it is nonetheless always wrong to murder.

${ }^{10}$ I have defended (2019: 2097-2100) the claim that 'see' and similar predicates are either assessmentinsensitive or sensitive to a different parameter than 'know'. I doubt anyone would suggest that 'murder' or 'Olympian' are assessment-sensitive.
} 
plines is relevant to many philosophical debates (as naturalism claims), such evidence cannot alone settle any philosophical debate. Scientific claims, for example, take their place as only one kind of data in such debates, and they are not necessarily the most important or the most strongly established kind.[...] Resolving [philosophical] debates involves weighing up many kinds of data and many methodological considerations by means of an often protracted and difficult cost-benefit analysis. (Daly and Liggins 2011: 322).

Let us say that the methodological priority of some factor is the importance assigned to that factor relative to other factors when deciding whether one theory is preferable to another. Adopting Daly and Liggins' terminology, let us say that the methodological priority is deferentialist if it permit semantics to settle philosophical theories. Conversely, the methodological priority is anti-deferentialist if it does not permit semantics to settle philosophical theories.

Anti-deferentialist methodological priority is at work in many classic philosophical arguments. For instance, here is one part of Kripke's famous attack on the Russellian view that proper names are disguised definite descriptions. According to this view, the name 'Aristotle' is a conjunction or inclusive disjunction of expressions like 'the teacher of Alexander', 'the author of the Posterior Analytics' and so on.

There is a certain theory, perhaps popular in some views of the philosophy of history, which might both be deterministic and yet at the same time assign a great role to the individual in history. Perhaps Carlyle would associate with the meaning of the name of a great man his achievements. According to such a view it will be necessary, once a certain individual is born, that he is destined to perform various great tasks and so it will be part of the very nature of Aristotle that he should have produced ideas which had a great influence on the western world. Whatever the merits of such a view may be as a view of history or the nature of great men, it does not seem that it should be trivially true on the basis of a theory of proper names. (Kripke 1981: 74-75, my emphasis).

We can agree then that, whether we should ultimately adopt deferentialist or anti-deferentialist methodological priority, anti-deferentialist methodological priority has at least substantial prima facie support. Moreover, we can also note that semantic relativists do not, at least to the best of my knowledge, have an explicit position on the matter. That is, they have not explicitly endorsed either deferentialist or anti-deferentialist methodological priority. We can therefore conclude that, if semantic relativism implies a commitment to deferentialist methodological priority, then semantic relativists owe us at least a prima facie acknowledgement and defence of this position. ${ }^{11}$

\footnotetext{
11 I have received mixed responses to anti-deferentialist methodological priority when I have presented this paper; some people endorse it, while others think it is far too strong. It is worth noting three things in response. First, following Daly and Liggins (2011), it is difficult to come up with reasons for denying anti-deferentialist methodological priority that do not ignore the distinction between non-philosophical considerations outright settling and merely informing philosophical debates. I suspect that at least some of the people who believe that anti-deferentialist methodological priority is too strong also believe that this view somehow entails a complete disregard for non-philosophical considerations, which is not the
} 
Most of the examples in Sect. 4 show that semantic relativism does imply a commitment to deferentialist methodological priority, at least in certain debates. Perception Pair falls under the ways of knowing claim, in the sense that it follows from the ways of knowing claim that PER 1 logically implies $\mathrm{PER}_{2}$. The ways of knowing claim is an epistemological position (e.g., Chisholm 1989: 41; Moore 1953: 77; Russell 1948: 422). Memory Pair falls under the more general epistemological claim that any factive state logically implies knowledge (Williamson 2000: 34), in the sense that it follows from this claim that $\mathrm{MEM}_{1}$ logically implies $\mathrm{MEM}_{2}$. And Morality Pair falls under at least some natural rights theories in ethics (see Wenar 2015: Section 6.1), in the sense that it follows from these theories that MORAL logically implies $\mathrm{MORAL}_{2}$.

Of course, it might be that we already reject, or ultimately would like to reject, these ways of knowing epistemologies or natural rights ethics. We might consider our best philosophy, including our best epistemology and ethics, and semantics jointly, and ultimately decide to adopt relativism about 'know' and 'wrong'. Or we might have already rejected ways of knowing and natural rights for other reasons (e.g. we are staunch sceptical nihilists, who believe in nothing), so the consequences of relativism about 'know' and 'wrong' do not bother us. But in either case the choice to opt for relativism would not be a deferentialist one-we would be opting for relativism as a result of a holistic consideration of our philosophy and semantics, fully cognizant of the consequences of relativism. The problem of deferentialism here is that, if we are relativists about expressions like 'know' and 'wrong', we do not get a choice in the matter; relativism has already settled our epistemological and ethical positions here for us. We adopted a certain view in semantics, but did not prepare for unforeseen consequences in our broader philosophy.

One response to this worry is to emphasise the distinction between, as it were, the business of philosophy and the business of semantics, and to urge that the relationship of logical implication in pairs like Perception, Memory, and Morality is the business of semantics rather than philosophy. As we noted in Sect. 4, one of the primary motivations for relativism is its ability to account for our intuitions about sentences, and this is widely considered to be the domain of semantics more generally (see, e.g., Tarasov 2018: 421-23). We also saw in Sect. 4 that relativism does not respect speaker intuitions about logical implication for pairs of propositions like Perception, Memory, and Morality. The response grants that this may be a problem, but insists that this is the full extent of the problem. Since accounting for speaker

\footnotetext{
Footnote 11 (continued)

case. Second, our arguments would still work with a weak version of anti-deferentialist methodological priority, viz. we have weak anti-deferentialist methodological priority if we do not permit semantics to settle substantive philosophical theories. However we ultimately define the criteria for being substantive, I think it is natural to view, e.g., the ways of knowing claim as substantive. Therefore, they would fit the bill even if we were to appeal to weak anti-deferential methodological priority. Finally, notice that I do not endorse either deferentialist or anti-deferentialist methodological priority here. It is enough for our dialectic that these views are put on the table in discussions of semantic relativism, and that it is recognised that semantic relativism carries an implied commitment to deferentialist methodological priority. These are heretofore unrecognised considerations in discussions of semantic relativism that deserve further examination.
} 
intuitions is the domain of semantics, accounting for speaker intuitions about logical implication in Perception Pair and others is a fortiori the domain of semantics. Moreover, these intuitions do not touch directly on the philosophical nature of the matters at hand. In short, there is not a deferentialist problem here because semantic relativism is doing only what semantics is supposed to do, and does not reach into the domain of philosophy. ${ }^{12}$

I will begin with a relatively simple counter-argument and conclude this section with a suggestion for a more ambitious methodological programme. The upshot of the simple counter-argument is that speaker intuitions $d o$ touch on the philosophical nature of the matters at hand, so there is not an easy way to confine the issues raised here to semantics only. The upshot of the more ambitious programme is that semantics and philosophy are linked directly, so the issues cannot be confined to one area.

The simple counter-argument starts with an intuition pump: there is an intuitive way of looking at Perception Pair, Morality Pair and others that links them with epistemological and ethical issues. Take the propositions $\mathrm{MORAL}_{1}$ and MORAL 2 again. I have the intuition that MORAL $\mathrm{M}_{1}$ implies MORAL $\mathrm{M}_{2}$. What accounts for this intuition? If the account is exclusively semantic, then it is something like I have the intuition because $\mathrm{MORAL}_{2}$ is true at every circumstance of evaluation at which MORAL $_{1}$ is true at. To be sure, this is part of the account, but it does not seem to be the whole account. If someone asks me why I have this intuition, I take it they are interested in whether I have a belief that gives rise to it. And if I can articulate this belief, I might say that there is a natural right to life for all persons and that violating that right amounts to doing something wrong. The full account then is something like I have the intuition because $\mathrm{MORAL}_{2}$ is true at every circumstance of evaluation at which MORAL ${ }_{1}$ is true at, and I believe that violating the natural right to life amounts to doing something wrong. The latter part of the account is philosophical in nature; it falls under the ethical theory of natural rights and is susceptible to philosophical attack and defence (see Wenar 2015: Section 6.1). If I now adopt semantic relativism about 'wrong', then my overall belief system (in this area) will be brought into incoherence. We have seen that $\mathrm{MORAL}_{1}$ fails to logically imply $\mathrm{MORAL}_{2}$ on this view, so either I cannot adopt relativism about 'wrong' after all, or I need to say that my intuition that $\mathrm{MORAL}_{1}$ logically implies $\mathrm{MORAL}_{2}$ and my belief that killing is a violation of rights and violating rights is wrong are in error.

We should now be in a position to put the point more directly: there is a clear route from our semantics for expressions like 'know' and 'wrong' to our philosophy, as demonstrated by the fact that semantic commitments can be shown to conflict with philosophical commitments. This suggestion gets further support from the broader debate about the semantics of 'know'. According to semantic contextualism, 'know' makes a different contribution to propositional content in different contexts, and therefore a sentence like 'Danny knows that Humpty Dumpty had a fall' expresses different propositions in different contexts (e.g. DeRose 2009: 47-66). One of the main motivations for contextualism about 'know' is its apparent ability to deal with external world scepticism. In his "Solving the Skeptical Problem" (1995),

${ }^{12} \mathrm{I}$ am grateful to an anonymous reviewer for suggesting this response. 
Keith DeRose famously argues that sceptical conclusions are compelling because they are true in certain contexts, but we do not need to worry about them because they are false in others. This is explained by the contextualist claim that 'know' makes a different contribution in different contexts to the propositional content of sceptical conclusions. External world scepticism is one of the quintessential epistemological problems, so by accounting for it using a semantic thesis DeRose exemplifies just the kind of route between semantics and philosophy that we are claiming here.

The more ambitious methodological programme begins with the premise that expressions like 'know' and 'wrong' pick out certain relations and properties in the world, and while it is the business of semantics to account for our intuitions about these expressions and the sentences that contain them, it is the business of philosophy to give us a metaphysics and epistemology for these relations and properties. For instance, semantics tells us the extension of 'wrong' and philosophy tells us what wrong is. But one cannot determine the full extension of 'wrong' without knowing what wrong is, and vice versa - my intuition about Moral Pair would be different if I had a different philosophy, and the intuition I do have tells me something about my philosophy. So there is a push-and-pull between semantics and philosophy here; the two cannot be carried out in isolation from each other.

Unlike the simpler argument above, which appeals to a route from semantics to philosophy, and vice versa, this premise establishes a direct relation between them. It is therefore controversial, and requires much more space than I have here to develop properly (see Tarasov 2014: Ch. 1).

\section{Unexpected Relativism}

In Sect. 3.3 we said that $\mathrm{p}$ fails to logically imply $\mathrm{q}$ if either condition $\mathrm{C}$ or D is satisfied, i.e. (C) p is expressed by an assessment-insensitive and $q$ is expressed by an assessment-sensitive sentence; or (D) p and q are expressed by assessment-sensitive sentences, but $\mathrm{p}$ and $\mathrm{q}$ are assessment-sensitive with respect to different parameters of circumstances of evaluation and the values of these parameters vary independently of each other. Notice that this is a sufficiency claim, so there may, of course, be other conditions under which p fails to logically imply q. Nonetheless, relativists might try to avoid the consequences highlighted in Sect. 4and 5 by arguing that conditions $\mathrm{C}$ and $\mathrm{D}$ are rarely, if ever, satisfied.

I have argued at length (2019: 2094-2100) that there is no convincing way for relativists about 'know' to get around at least one of conditions $\mathrm{C}$ or D being satisfied. I started from the assumption that perception sentences, i.e. $\left\ulcorner_{\mathrm{X}}\right.$ perceives that $\Phi\urcorner$, are assessment-insensitive and knowledge attributions, i.e. $\ulcorner\mathrm{x}$ knows that $\Phi\urcorner$, are assessment-sensitive. This fulfils condition (1) above, and thus the proposition expressed by the former does not logically imply the proposition expressed by the latter (Tarasov 2019: 2094-97). I then acknowledged that, faced with this consequence, relativists would naturally claim that perception sentences are also assessment-sensitive. The expectation is that, by rendering both the sentence that expresses the logically implying proposition and the sentence that expresses the 
logically implied proposition assessment-sensitive, the relation of logical implication between the two propositions may be preserved (Tarasov 2019: 2097).

We already know that, following condition D, this expectation fails if the two sentences are assessment-sensitive with respect to different parameters of circumstances of evaluation and the values of these parameters vary independently of each other. And I have argued (2019: 2097-100) that this is precisely what happens with perception sentences and knowledge attributions. Be that as it may, in this section we are interested in the dialectical move from the claim that one type of sentence, e.g. knowledge attributions, is assessment-sensitive, to the claim that two types of sentences, e.g. knowledge attributions and perception sentences, are assessmentsensitive. We are interested in this move because it suggests something potentially worrying about semantic relativism, viz. that in order to avoid controversial philosophical consequences or deferentialist methodological priority, relativists have to radically expand the reach of their theory. For instance, whereas one might have started off only as a relativist about the predicate 'knows', following my arguments one finds that one also needs to be a relativist about predicates like 'sees', 'hears' and others. More generally, if a set of propositions Q is logically implied by a set of propositions $\mathrm{P}$, which is in turn logically implied by a set $\mathrm{R}$ and so on, and if one wants to say that the propositions that are members of $\mathrm{Q}$ are expressed by assessment-sensitive sentences, one also needs to say that the members of $\mathrm{P}, \mathrm{R}$ and so on are expressed by assessment-sensitive sentences.

This chain of logical implication relations can be reasonably easy to get underway. For example, consider the following three propositions:

$\mathrm{PER}_{3}$ : that Danny underwent some relevant physical process $\mathrm{x}$.

PER $_{1}$ : that Danny saw that Humpty Dumpty had a fall.

$\mathrm{PER}_{2}$ : that Danny knows that Humpty Dumpty had a fall.

We might start with the claim that only knowledge attributions are assessment-sensitive. But in this case we would find that $\mathrm{PER}_{1}$ fails to logically imply $\mathrm{PER}_{2}$. If we wanted to preserve this relation, we would need to expand the set of assessmentsensitive sentences to include perception sentences. Then, if we thought that $\mathrm{PER}_{3}$ is expressed by an assessment-insensitive sentence, we would in turn find that PER $_{3}$ fails to logically imply $\mathrm{PER}_{1}$. Depending on our theory of perception - in particular, depending on whether we think perceiving something is a matter of undergoing some relevant physical process - we might want to say that $\mathrm{PER}_{3}$ does logically imply $\mathrm{PER}_{1} \cdot{ }^{13}$ Thus, we would need to expand the set of assessment-sensitive

\footnotetext{
${ }^{13}$ The original formulation of $\mathrm{PER}_{3}$ did not have the qualifier 'relevant'. I am grateful to an anonymous reviewer for pointing out that without a qualifier like this it is trivially false that $\mathrm{PER}_{3}$ logically implies $\mathrm{PER}_{1}$. For instance, as the reviewer suggests, if Danny closed his eyes, he underwent a physical process, but then obviously he did not see that Humpty Dumpty had a fall. The current formulation is not ideal, because 'relevant' is likely a context-sensitive or a context-relative term, but it is the most concise way of qualifying $\mathrm{PER}_{3}$. The idea is simply that 'some relevant physical process $\mathrm{x}$ ' is a placeholder for our best description of the physics and biology of perception.
} 
sentences again, to include not only knowledge attributions and perception sentences, but what we might call physical process sentences. Presumably, this would be a lot more relativism than we originally bargained for-what we might call the problem of unexpected relativism. ${ }^{14}$

Commitment to unexpected relativism may be prevented in one of two ways. First, we might find propositions that stand in the relation of logical implication to relevant others but the sentences that express them do not meet condition $\mathrm{D}$, i.e. we might find that $\mathrm{p}$ and $\mathrm{q}$ are expressed by assessment-sensitive sentences, but $\mathrm{p}$ and $\mathrm{q}$ are not assessment-sensitive with respect to different parameters of circumstances of evaluation. Or second, we might eventually come up against sentences that are implausible candidates for being assessment-sensitive.

The last route is little consolation to relativists: if we eventually come up against sentences that are implausible candidates for being assessment-sensitive, we will find ourselves in a situation that meets condition $\mathrm{C}$, and thus one in which the relevant propositions fail to logically imply relevant others. For instance, arguably it is implausible that the sentence 'Danny underwent some relevant physical process $\mathrm{x}$ ' is assessment-sensitive. However, if this sentence expresses $\mathrm{PER}_{3}$ and perception sentences are assessment-sensitive, then we have a situation that satisfies condition $\mathrm{C}$ and thus $\mathrm{PER}_{3}$ does not logically imply $\mathrm{PER}_{1}$. In other words, this way of avoiding unexpected relativism comes at the expense of deferentialist methodological priority; we are back to the issue we raised in Sect. 5 .

The view that there are propositions that do stand in the relation of logical implication to relevant others but the sentences that express them do not meet condition (2) is a promising one. However, the truth of this view would need to be established case by case. For instance, we have already seen that, following my earlier arguments, this view is implausible as far as perception sentences and knowledge attributions are concerned; these sentences do appear to meet conditions C and D. We will need to perform similar examinations for other types of sentences. For example, even if it can be plausibly argued $\mathrm{MORAL}_{1}$ is expressed by an assessment-sensitive sentence, there remains the further question of whether or not the sentences that express $\mathrm{MORAL}_{1}$ and $\mathrm{MORAL}_{2}$ fall under condition D.

To sum up, our observations in this section suggest that relativism about some expressions can quite easily lead to relativism about some other expressions, which can be an unexpected and potentially unwanted result. Semantic relativists can resist this by examining each relevant pair of logically implying and logically implied propositions to determine that this pair does not fall under conditions $\mathrm{C}$ or $\mathrm{D}$. In this case the onus is placed on relativists to pick up the argument.

\footnotetext{
14 I am extremely grateful to an anonymous reviewer for pressing me to reformulate my argument here. A previous draft was more ambitious, but far less plausible. I owe the term 'unexpected relativism' to the reviewer.
} 


\section{Can Relativist Semantics be Modified to Avoid the Problem?}

We have established that $\mathrm{p}$ fails to logically imply $\mathrm{q}$ when conditions $\mathrm{A}$ and $\mathrm{B}$ are met; roughly put, when the truth-values of $p$ and $q$ vary with respect to different parameters in a particular way. So it is natural to ask whether we can avoid conditions A and B altogether; specifically, whether we can modify relativist semantics in such a way that conditions A and B are never met.

The most obvious approach is to render relativist semantics more coarse-grained. That is, since the issues above are caused by the truth-values of $p$ and $q$ varying with respect to different parameters, the obvious solution is to replace the different parameters with a single parameter. To see this, recall §3.2. There we characterised circumstances of evaluation as sequences of a world $w$ and two unorthodox parameters $n$ and $o:<w, n, o>$. We saw that when $p$ is sensitive to changes to $w$ and $n$ but not $\mathrm{o}$, and $\mathrm{q}$ is sensitive to changes in $\mathrm{w}$ and o but not $\mathrm{n}, \mathrm{p}$ fails to logically imply $\mathrm{q}$. So the solution is to replace both $\mathrm{n}$ and $\mathrm{o}$ with what we might call a master parameter $m$, which ranges over all the values that $n$ and o range over. Parameters like $n$ and o stand for epistemic standards, standards of taste, moral standards and others, so we need to assume that $m$ ranges over all of these.

As with any coarse-grained approach, there is an obvious price: this modification would make relativist semantics less expressive than it is currently. We would no longer be able to say that knowledge attributions are assessment-sensitive with respect to epistemic standards, that some sentences are assessment-sensitive with respect to moral standards, and so on. Instead, we would have to say that all assessment-sensitive sentences are assessment-sensitive in the same way, i.e. that they are all assessment-sensitive with respect to the master parameter. Of course, informally we could continue to make distinctions between sentences that are sensitive to different kinds of standards, but these distinctions would no longer be recognised within the relativist semantics.

A more significant problem is that this approach offers at best only a partial solution to the issues above. Recall that $\mathrm{p}$ also fails to logically imply $\mathrm{q}$ when $\mathrm{p}$ is sensitive to changes to $\mathrm{w}$ but not $\mathrm{n}$, and $\mathrm{q}$ is sensitive to changes to $\mathrm{w}$ and $\mathrm{n}$, which is the case when $\mathrm{p}$ is expressed by an assessment-insensitive sentence and $\mathrm{q}$ by an assessment-sensitive sentence. While it might be at least prima facie plausible to replace the unorthodox parameters $\mathrm{n}, \mathrm{o}$ and others with a single parameter $\mathrm{m}$, it is far less plausible to replace the possible worlds parameter w with $\mathrm{m}$. It would transform semantic relativism from an extension of possible worlds semantics to a framework that subsumes possible worlds semantics. Moreover, this approach would be even more coarse-grained than the one above; $m$ alone would capture all the values that were previously captured by a long sequence of parameters without any differentiation between the types of values or how they are assigned.

In short, we should reject any further extensions to the master parameter approach. But then the approach allows us to avoid conditions A and B only where both $\mathrm{p}$ and $\mathrm{q}$ are expressed by assessment-sensitive sentences, where what is at issue is the interaction between the values of parameters like $n$ and o. The modification is of no use where $\mathrm{p}$ is expressed by an assessment-insensitive sentence and $\mathrm{q}$ is expressed by an assessment-sensitive sentence, where what is at issue is 
the interaction between the values of $\mathrm{w}$ and parameters like $\mathrm{n} .{ }^{15}$ This means that the modification resolves the issues above only to the extent that we think that both propositions like $\mathrm{PER}_{1}$ and propositions like $\mathrm{PER}_{2}$ are expressed by assessmentsensitive sentences. If we think that $\mathrm{PER}_{1}, \mathrm{MORAL}_{1}$ and others are expressed by assessment-insensitive sentences, then we must still deal with the issues raised in Sects. 4 and 6.

\section{Conclusion}

We have seen that my 2019 claims about semantic relativism about 'know' generalise to semantic relativism more broadly and we noted some worrying consequences of this generalisation. In particular, we noted that semantic relativism ends up being incompatible with more than just the ways of knowing claim, that it implies a commitment to deferentialist methodological priority and that it invites what we have called unexpected relativism. We also saw that the natural way to modify relativist semantics would at best avoid only some of these issues at the price of a more coarse-grained semantics. While there are prima facie reasons to be concerned about these consequences, the extent to which we ultimately find them worrying is open to debate. Nonetheless, the fact that these consequences are there to be debated is, to the best of my knowledge, a novel finding. Semantic relativism is a view that raises more philosophical questions than relativists and non-relativists alike have accounted for. Whichever way these questions are ultimately answered, we have a new line of enquiry to engage semantic relativists on, and in the broader discussions of the semantics of expressions like 'know', 'wrong' and others.

Acknowledgements I would like to thank several anonymous reviewers for their input. One anonymous reviewer in particular has provided so much technical insight and encouragement, including the proofs in the Appendix, that in my view they have co-authored this paper (unfortunately I haven't been able to find out their name to properly credit their work). I am grateful to Martina Faller, Andrew Koontz-Garboden, Graham Stevens, and the members of the Semantics Lab at the University of Manchester for comments on earlier drafts. Thanks to Robert Knowles and Jack Winter for early discussions of and around these topics. Thanks also to Rob, Andrew Kirton and Pamela Donnellan for suggestions for later drafts. Finally, thanks to everyone, and especially to Thomas Uebel, who contributed to "Semantic Relativism and Ways of Knowing", which lays the foundations for many of the arguments in this paper.

Open Access This article is licensed under a Creative Commons Attribution 4.0 International License, which permits use, sharing, adaptation, distribution and reproduction in any medium or format, as long as you give appropriate credit to the original author(s) and the source, provide a link to the Creative Commons licence, and indicate if changes were made. The images or other third party material in this article are included in the article's Creative Commons licence, unless indicated otherwise in a credit line to the material. If material is not included in the article's Creative Commons licence and your intended use is not permitted by statutory regulation or exceeds the permitted use, you will need to obtain permission directly from the copyright holder. To view a copy of this licence, visit http://creativecommons.org/licen ses/by/4.0/.

\footnotetext{
15 To see this, just swap the master parameter $\mathrm{m}$ for $\mathrm{n}$ in our examples in $\S 3.1$.
} 


\section{Appendix: Proofs}

The demonstrations in the main text above use a toy semantic model that has only a handful of circumstances of evaluation and makes certain assumptions about the truth of $\mathrm{p}$ and $\mathrm{q}$. The proofs below are offered to show that my arguments in those sections are quite general. ${ }^{16}$

Suppose that $\mathrm{p}$ is expressed by a contingent $A I S$ sentence $\Phi$. Suppose $\mathrm{q}$ is expressed by a contingent $A S$ sentence $\Psi$. For shorthand, say $\mathrm{p}$ is contingent and $A I S$; $\mathrm{q}$ is contingent and $A S$. Say that a proposition is $A S$ to a parameter if its truthvalue varies with respect to changes in that parameter. Say that a proposition is $A I S$ to a parameter if its truth-value does not vary with respect to changes in that parameter. Call a circumstance of evaluation a p-circumstance if $\mathrm{p}$ is true at this circumstance. Call a circumstance of evaluation a q-circumstance if $q$ is true at this circumstance. Let the semantic turnstile ' $\models$ ' stand for Logical Implication. Then:

Proof that $p$ does not logically imply $q$ under condition (i), i.e. where $p$ is expressed by an assessment-insensitive and $q$ is expressed by an assessment-sensitive sentence:

By AIS and the contingency of $\mathrm{p}$, there must be at least one possible world $\mathrm{w}_{1}$ such that, for any value of $\mathrm{n},<\mathrm{w}_{1}, \mathrm{n}>\vDash \mathrm{p}$. Hold $\mathrm{w}_{1}$ fixed. Then, by $A S$ of $\mathrm{q}$, there must be at least one value $\mathrm{n}_{1}$ such that $\left\langle\mathrm{w}_{1}, \mathrm{n}_{1}>\vDash \mathrm{q}\right.$, and at least one other value $\mathrm{n}_{2}$ such that $<\mathrm{w}_{1}, \mathrm{n}_{2}>\not \models \mathrm{q}$. So there is a circumstance $<\mathrm{w}_{1}, \mathrm{n}_{2}>$ that is a $\mathrm{p}$-circumstance but not a $\mathrm{q}$-circumstance. Therefore, $\mathrm{p} \not \models \mathrm{q}$.

Proof that $p$ does not logically imply $q$ under condition (ii), i.e. where $p$ and $q$ are expressed by assessment-sensitive sentences, but $p$ and $q$ are assessment-sensitive with respect to different parameters of circumstances of evaluation and the values of these parameters vary independently of each other:

By $A S$ of $\mathrm{p}$ to $\mathrm{n}$, for all $\mathrm{w}$ and o, there is at least one value $\mathrm{n}_{1}$ and at least one other value $\mathrm{n}_{2}$ such that $<\mathrm{w}, \mathrm{n}_{1}, \mathrm{o}>\vDash \mathrm{p}$ and $<\mathrm{w}, \mathrm{n}_{2}, \mathrm{o}>\not \models \mathrm{p}$. By $A S$ of $\mathrm{q}$ to $\mathrm{o}$, for all $\mathrm{w}$ and $\mathrm{n}$, there is at least one value $\mathrm{o}_{1}$ and at least one other value $\mathrm{o}_{2}$ such that $<\mathrm{w}, \mathrm{n}, \mathrm{o}_{1}>\vDash$ $\mathrm{q}$ and $<\mathrm{w}, \mathrm{n}, \mathrm{o}_{2}>\not \models \mathrm{q}$. Let $<\mathrm{w}, \mathrm{n}_{1}, \mathrm{o}_{1}>\vDash \mathrm{p}$ and $<\mathrm{w}, \mathrm{n}_{1}, \mathrm{o}_{1}>\vDash \mathrm{q}$. Let $\mathrm{o}_{2}$ be such that $<\mathrm{w}, \mathrm{n}_{1}, \mathrm{o}_{2}>\not \models \mathrm{q}$. By the AIS of $\mathrm{p}$ to $\mathrm{o},<\mathrm{w}, \mathrm{n}_{1}, \mathrm{o}_{2}>\vDash \mathrm{p}$. So there is a circumstance $<\mathrm{w}_{1}, \mathrm{n}_{1}, \mathrm{o}_{2}>$ that is a p-circumstance but not a q-circumstance. Therefore, $\mathrm{p}$ $\not \models \mathrm{q}$.

\section{References}

Chisholm, R. (1989). Theory of knowledge (3rd ed.). NJ: Prentice-Hall Inc.

Daly, C., \& Liggins, D. (2011). Deferentialism. Philosophical Studies, 156(3), 321-337.

DeRose, K. (1995). Solving the skeptical problem. The Philosophical Review, 104(1), 1-52.

DeRose, K. (2009). The case for contextualism: knowledge, skepticism, and context (Vol. 1). Oxford: Oxford University Press.

\footnotetext{
${ }^{16}$ I am very grateful to an anonymous reviewer for re-assuring me that the core claims of this and my 2019 papers are sound, and for generously suggesting full proofs for this. (I hasten to add that, while the proofs above are very heavily based on the reviewer's, any remaining technical issues are my own.).
} 
Glanzberg, M. (2007). Context, content, and relativism. Philosophical Studies, 136(1), 1-29.

Kaplan, D. (1989). Demonstratives: An essay on the semantics, logic, metaphysics, and epistemology of demonstratives and other indexicals. In J. Almog, J. Perry, \& H. Wettstein (Eds.), Themes from Kaplan (pp. 481-563). Oxford: Oxford University Press.

Kölbel, M. (2008). Motivations for relativism. In M. García-Carpintero \& M. Kölbel (Eds.), Relative truth (pp. 1-38). Oxford: Oxford University Press.

Kripke, S. (1981). Naming and necessity. Oxford: Blackwell Publishing.

MacFarlane, J. (2014). Assessment sensitivity: relative truth and its applications. Oxford: Oxford University Press.

Moore, G. E. (1953). Some main problems of philosophy. London: George Allen \& Unwin Ltd.

Russell, B. (1948). Human knowledge: Its scope and limits. New York: Simon and Schuster.

Tarasov L (2014). The semantics of knowledge attributions: A defence of moderate invariantism. The University of Manchester: Manchester (PhD thesis).

Tarasov, L. (2018). Why we need corpus linguistics in intuition-based semantics. Grazer Philosophische Studien, 95(4), 421-435.

Tarasov, L. (2019). Semantic relativism and ways of knowing. Synthese, 196(5), 2089-2109.

Wenar, L. (2015). Rights. In E. Zalta (Ed.), The Stanford Encyclopedia of Philosophy. https://plato.stanf ord.edu/archives/fall2015/entries/rights/.

Williamson, T. (2000). Knowledge and its limits. Oxford: Oxford University Press.

Wright, C. (2008). Relativism about truth itself: Haphazard thoughts about the very idea. In M. GarcíaCarpintero \& M. Kölbel (Eds.), Relative truth (pp. 157-185). Oxford: Oxford University Press.

Publisher's Note Springer Nature remains neutral with regard to jurisdictional claims in published maps and institutional affiliations. 